\section{The bioentrepreneur's road map}

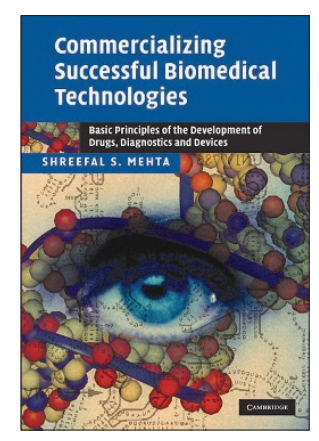

plan), (vi) production (manufacture) and (vii) profits (reimbursement). This framework allows the aspiring entrepreneur to systematically assess the components of a sound commercialization plan in a way that doesn't allow him or her to lose sight of the big picture, yet still manage the details required for successful commercialization.

Mehta wisely addresses the practical limitations of using a linear road map to organize the iterative and path-dependant process of biomedical product development. He points out, "The linear road map shows the components that must be assessed to build a sound commercialization plan, but the processes are all carried out in parallel, with shifting emphasis on each component as one proceeds down the plan." The text prepares the reader to organize the commercialization process while at the same time pointing out the inevitable fact that feedback from one component will ultimately influence or change the understanding of another previously researched component. For example, limited access to intellectual property rights may change market strategy, which in turn may alter the regulatory pathway required to develop an FDA-approved product.

Throughout, Mehta highlights the significant differences that are specific to biomedical technology commercialization as compared to the rest of the free-market industries in the United States. Most importantly, biomedical technologies are heavily regulated, requiring a significant commercialization planning to account for this issue. Another complexity not often faced by other technology sectors is that the user-that is, the patient—does not make the purchasing decision; it is made instead by a provider such as a physician or pharmacy benefit manager. Adding more complexity to the situation, the government or insurance company is the payer, requiring the entrepreneur to consider reimbursement strategies early on-or otherwise bear the burden of having a tremendous product with nobody willing to prescribe or pay for it. Factor in other issues ranging from what truly is a patentable invention in the life sciences to how to commercially manufacture products in accordance with current good manufacturing practices and you begin to see how much planning must go into bringing biomedical technologies to market.

As in many cases, the best way to allow readers to fully grasp a technology commercialization concept is to apply the concepts covered in the text to real-world technology examples. The text does include excellent case studies and excerpts throughout; however, the reader will crave more-an issue that will hopefully be addressed in subsequent editions and/or by supplementary materials.

Although there are many general technology commercialization materials available to students, researchers and entrepreneurs on the market, the advice given often lacks the context of the biomedical technology space-specifically the regulatory and reimbursement issues that significantly affect the planning required to bring biomedical technologies to market. Often, readers and teachers interested in biomedical technology commercialization are forced to supplement general technology commercialization materials with specific biomedical commercialization materials. Although no single text could possibly provide all the information necessary for an individual to translate biomedical technology research into a biomedical technology product, Mehta does an excellent job of identifying and organizing the major issues associated with biomedical technology commercialization in a framework that students, researchers and entrepreneurs can understand. 